\title{
Joint longitudinal and survival-cure models in tumour xenograft experiments
}

\author{
Jianxin Pan’ Yanchun Bao, $\stackrel{\ddagger}{\ddagger}$ Hongsheng Dai, $\stackrel{\S}{\S}$ Hong-Bin Fang, 9
}

\begin{abstract}
In tumour xenograft experiments, treatment regimens are administered and the tumour volume of each individual is measured repeatedly over time. Survival data are recorded due to the death of some individuals during the observation time period. Also, cure data are observed due to a portion of individuals who are completely cured in the experiments. When modelling these data, certain constraints have to be imposed on the parameters in the models to account for the intrinsic growth of the tumour in the absence of treatment. Also, the likely inherent association of longitudinal and survival-cure data has to be taken into account in order to obtain unbiased estimators of parameters. In this paper, we propose such models for the joint mod-
\end{abstract}

${ }^{*}$ School of Mathematics, The University of Manchester, Oxford Road, Manchester M13 9PL, UK

$\dagger$ Email: jianxin.pan@manchester.ac.uk

${ }^{\ddagger}$ School of Mathematics, The University of Manchester, Oxford Road, Manchester M13 9PL, UK

$\S$ Department of Mathematical Sciences, University of Essex

IDivision of Biostatistics, University of Maryland Greenbaum Cancer Center, Baltimore, MD 21201, USA 
elling of longitudinal and survival-cure data arising in xenograft experiments. Estimators of parameters in the joint models are obtained using a Markov chain Monte Carlo approach. Real data analysis of a xenograft experiment is carried out and simulation studies are also conducted, showing that the proposed joint modelling approach outperforms the separate modelling methods in the sense of mean squared errors.

Keywords: Constrained parameters; joint longitudinal and survival-cure model; Markov chain Monte Carlo; xenograft experiment. 


\section{Introduction}

In cancer drug development, demonstrating anti-tumor activity in an in vivo experiment is an important and necessary step to make a promising experimental treatment available to humans. The xenograft model is a commonly used in vivo model in cancer research, for which severe combined immunodeficient (scid) mice are grafted with human cancer cells after which they receive a treatment and are then followed up. Tan et al. [1] presented a typical xenograft experiment, where several treatment regimens are administered and an outcome variable, tumour volume, is measured at the start of the treatment and then at regular follow-up times. In the literature, methodology has been developed to analyze repeated measurements and survival times collected from xenograft experiments. For example, Tan et al. [2] developed a t-test via the EM algorithm and also a Bayesian approach for testing for differences in effects between two treatment regimens. If no treatment were given to the tumour-bearing mice, the tumours would keep growing until the mice died or are sacrificed. Therefore, certain constraints have to be imposed on the parameters in the model to account for the intrinsic growth of a tumour in the absence of treatment. Tan et al. [1] considered a class of regression models for longitudinal outcomes with constrained parameters. Fang et al. [3] proposed a Bayesian hierarchical model to account for the parameter constraints. However, these authors ignored the very likely inherent association between longitudinal responses and survival outcomes for data coming from the same subject. As a result, such statistical inferences may 
be biased.

Joint models for both longitudinal and survival data have been developed in recent years and are extensively reviewed by [4]. It is well known nowadays that analyzing combined longitudinal data and survival data can lead to a significant improvement in the efficiency of statistical modelling compared to the separate analyses - see, for example, [5], [6], [7], [8], [9], [10], [11], [12] and [13]. When there are cured individuals in the survival studies, either due to immunes or long-term survivors, joint models for survival and cured data have been considered by [14], [15], [16] and [17]. Longitudinal and survival-cure models have been further developed by [18], [19] and [20]. The mixture distribution for the longitudinal model in [18] is very unique which is not suitable to our study. Yu and his colleagues $[19,20]$ studied a prostate cancer data, where longitudinal data have an inherent relationship with survival outcomes and survival hazard rate are modelled separately for cured patients and uncured patients. However, these models are not suitable for analyzing the tumour data arising in xenograft experiments for the following reason. This is because these models assume the cure probability only depends on the baseline covariates. This assumption may not be true in xenograft experiments because the cure probability of a mouse will clearly be related not only to the baseline tumor size but also to how fast the tumor grows or other characteristics of tumor volumes.

In this paper, motivated by an xenograft experiment for mice, we propose new joint models for longitudinal and survival-cure data by taking into account 
not only the likely inherent association of the different types of data but also the intrinsic growth of a tumour in the absence of any treatment [21]. The random effects in linear mixed models for longitudinal data, after being properly scaled, are incorporated into the Cox hazard model for survival data and the logistic model for cure data, so that the inherent association between the different types of data can be accounted for. The fixed effects in the longitudinal linear mixed models, on the other hand, are imposed constraints in order to account for the intrinsic growth of a tumour in the absence of treatment. Posterior inferences for the parameters in the models are obtained by using a Markov chain Monte Carlo (MCMC) method.

The rest of the paper is organized as follows. In Section 2, we define the joint longitudinal and survival-cure models for the xenograft experimental data and provide the log-likelihood function for the complete data. In Section 3, we specify prior distributions for the parameters and provide model selection criteria for finding the best model. In Section 4, we use an MCMC method to generate random samples from the posterior distributions of the parameters and apply to the real xenograft data analysis involving two new anticancer agents against rhabdomyosarcomas. In Section 5 we carry out simulation studies to assess the performance of the proposed approach. Numerical results show that the proposed joint modelling strategy outperforms the separate modelling methods. Further comments and discussions are given in Section 6. 


\section{Data and Models}

\section{$2.1 \quad$ Data set}

The xenograft experimental data in [1] and [24] are about two new anticancer agents: temozolomide (TMZ) and irinotecan (CPT-11). TMZ is a methylating agent that has been approved for treatment of astrocytoma and is entering various phases of clinical evaluation against tumours. CPT-11 has demonstrated a broad activity against both murine and human tumour xenograft models and clinically significant activity against many types of cancer. A DNA analysis has formed the biochemical rationale for combining TMZ and CPT-11. Our primary objective is to analyze the activity of TMZ combined with CPT-11 against one rhabdomyosarcoma (Rh18) xenograft. Mice from the same strain were used and they are virtually genetically identical. In total, we have 51 subjects (mice) observed, which are divided into eight groups for different treatment regimens. Two dose levels were considered for both anticancer agents; for TMZ the two weekly dose levels considered are $140 \mathrm{mg} / \mathrm{kg}$ and $210 \mathrm{mg} / \mathrm{kg}$; for CPT-11 the two levels are $2.0 \mathrm{mg} / \mathrm{kg}$ or $3.05 \mathrm{mg} / \mathrm{kg}$. Tumor-bearing mice were treated under certain levels of either TMZ, CPT-11 or both. Note that the mice were treated on a one-week or two-week courses per three-week cycle. Table 1 in the Supplementary Web Materials provides more detailed information.

The tumour volume was measured at the initial time and once a week within the follow-up period of 12 weeks. Figure 1 shows the change of tumor volume 
$\left(\mathrm{cm}^{3}\right)$ with time for mice in each of the eight treatment groups.

[Figure 1 about here.]

From Figure 1, it is clear that in the control group (i.e., no treatment) the tumor volume increases with time, while in other treatment groups the tumor volume may decrease in the beginning and then increase at later times. Figure 1 displays the longitudinal measurements observed until mice died or were sacrificed due to the tumour volume quadrupled. Among these 51 mice, in total 25 mice either died of toxicity or were sacrificed and the remaining 26 mice survived longer than the 12-week observation period. For these survived ones, their lifetimes cannot be observed but were censored at the end of 12 weeks. On the other hand, 14 mice quickly shrank their tumour volumes smaller than $0.01 \mathrm{~cm}^{3}$, which became too small to be observable by a reading machine, and had no recurrent growth of tumour in the rest period of the experiment. For this portion of mice, it is believed that they are very likely cured already, see [1] for more details. We also note that a few of the mice had the tumour disappear $\left(<0.01 \mathrm{~cm}^{3}\right)$ first but grow back in later weeks up to the end of the experiment. These mice cannot be considered as cured ones but the intermittent missing values are truncated as $0.01 \mathrm{~cm}^{3}$. We are therefore motivated by this dataset to build longitudinal models for repeated measurements of the tumour volume, survival models for time-to-death or sacrifice of the mice, and cure models for the cured mice, simultaneously. 


\subsection{Longitudinal data sub-model}

Consider in general the anti-tumour activity of $S$ agents. Suppose that there are $n+1$ pre-specified follow-up times $t_{0}<t_{1}<\cdots<t_{n}$ for each of the $m$ subjects/mice. Let $Y_{i}\left(t_{j}\right)$ be the tumour volume of the $i$ th mouse measured at the time $t_{j}(j=0,1, \cdots, n)$. To make the data normally distributed, we assume that a $\log$ scale has been introduced to $Y_{i}\left(t_{j}\right)$. Denote $x_{i}^{(s)}(t)$ $(s=1, \cdots, r)$ as the cumulative dose of the $s$ th agent (or interactions of two agents) administered to the $i$ th mouse until the time $t$.

The responses $Y_{i}\left(t_{j}\right)\left(j=1, \ldots, n_{i}\right.$ and $\left.i=1, \ldots, m\right)$ may be modelled by a linear mixed model [22]

$$
Y_{i}\left(t_{j}\right)=\psi_{0}+\psi_{1} t_{j}+\psi_{2} t_{j}^{2}+\boldsymbol{X}_{i}\left(t_{j}\right) \boldsymbol{\beta}+u_{i 0}+u_{i 1} t_{j}+\varepsilon_{i j}
$$

where the random effect $\boldsymbol{u}_{i}=\left(u_{i 0}, u_{i 1}\right)^{\prime}$ follow a normal distribution $N_{2}\left(\mathbf{0}, \Sigma_{u}\right)$, $\boldsymbol{\psi}=\left(\psi_{0}, \psi_{1}, \psi_{2}\right)$ and $\boldsymbol{\beta}=\left(\beta_{0}, \ldots, \beta_{r}\right)^{\prime}$ are unknown parameter vectors, $\boldsymbol{X}_{i}\left(t_{j}\right)=$ $\left(x_{i}^{(1)}\left(t_{j}\right), \ldots, x_{i}^{(r)}\left(t_{j}\right)\right)$, and random errors $\varepsilon_{i j}$ are independent and normally distributed with $E\left(\varepsilon_{i j}\right)=0$ and $\operatorname{Var}\left(\varepsilon_{i j}\right)=\sigma_{\varepsilon}^{2}$.

Here we consider such a simple quadratic baseline tumor growth model $\psi_{0}+\psi_{1} t_{j}+\psi_{2} t_{j}^{2}$, because the plot of log-tumor size against time shows a quadratic trajectory, see Figure 1 of the Supplementary Web Materials. For simplicity we consider a simple linear random effects model $u_{i 0}+u_{i 1} t$ to capture the within-subject correlation between the repeated measures of the

tumour volume. Note that the models can be extended to more complicated 
tumor growth models [23] involving more random effects.

As pointed out by [1], in the xenograft experiments the tumour born by an immunosuppressants mouse in the control group is expected to grow over the follow-up period. Ignoring this fact can lead to misleading inferences,

for example, resulting in underestimates of treatment effects [1]. In order to reflect this fact in the model, $\boldsymbol{\psi}$ is assumed to be such that,

Condition 1: The quadratic polynomial in time

$$
\psi_{0}+\psi_{1} t+\psi_{2} t^{2}
$$

is an increasing function for $t>0$.

As long as $\psi_{1}+2 \psi_{2} t>0$ where $t>0$, Condition 1 above holds. A special case is a straight line with $\psi_{2}=0$ and $\psi_{1}>0$. We denote $\boldsymbol{\Psi}=\{\boldsymbol{\psi}$ : such that $\left.\psi_{1}+2 \psi_{2} t>0\right\}$. In other words, $\boldsymbol{\Psi}$ is the collection of all possible values of $\psi_{1}$ and $\psi_{2}$ such that the quadratic polynomial in (2) is an increasing function.

\subsection{Proportional hazard and cure sub-models}

Note that we cannot justify whether a mouse is really cured or not, since when tumor size is less than $0.01 \mathrm{~cm}^{3}$ it can not be measured and we do not know whether the tumor really disappear or not. It happened in several mice that the tumor size became smaller than 0.01 and reoccur later on. In practice, the experiment researchers usually assume that if the tumor size is 
less than 0.01 at week 12, the end of the experiment, then the tumor will never reappear, i.e. a mouse is 'cured' eventually.

Let $\xi_{i}=0$ denote the $i$ th mouse 'cured' by agents and $\xi_{i}=1$ be not 'cured'. Assume $p_{i}=\operatorname{Pr}\left(\xi_{i}=1\right)$, the probability of incidence that the event, death caused by the tumour problem or toxicity of agents, occurs by the end of the experiment. Obviously, a cured mouse does not experience the death or sacrifice in the experiment period. Conversely, a mouse who died or was sacrificed during the experiment period must have the incidence $\xi_{i}=1$. In addition, we may not know whether a mouse is cured or not, if the tumor is larger than 0.01 by the end of the experiment.

Therefore it is clear that the binary incidence indicator $\xi$ will have three possibilities: (a) when the censoring occurs $\left(\delta_{i}=0\right)$, the incidence indicator $\xi_{i}$ will be unobserved if the tumor size at the censoring time, i.e., week 12 , is larger than 0.01 ; (b) when the censoring occurs $\left(\delta_{i}=0\right)$ and the tumor size at the censoring time is not more than 0.01 at week 12 . That is, the tumor almost disappears by the end of the experiment, so that it can be treated as $\xi_{i}=0$ (cured); (c) the censoring does not occur, i.e., the failure indicator is $\delta_{i}=1$, so that $\xi_{i}=1$ (not cured).

Let $T_{i}$ be the time to death for the $i$ th individual, defined only for those with $\xi_{i}=1$, with the hazard function $h\left(t \mid \xi_{i}=1\right)$ and the survival function $S\left(t \mid \xi_{i}=1\right)$. If a mouse survives longer than the experiment period, the survival time of the mouse is censored as the mouse is either cured or has no enough follow-up times. In other words, we actually observe $\tilde{T}_{i}=\min \left(T_{i}, C_{i}\right)$ 
and $\delta_{i}=I\left(T_{i} \leq C_{i}\right)$ where $C_{i}$ is the censoring time and $\delta_{i}$ is the relative failure indicator.

The marginal survival function of $T_{i}$ therefore is given by

$$
\begin{aligned}
S_{i}(t) & =\operatorname{Pr}\left(T_{i} \geq t\right) \\
& =\operatorname{Pr}\left(T_{i} \geq t \mid \xi_{i}=0\right) \operatorname{Pr}\left(\xi_{i}=0\right)+\operatorname{Pr}\left(T_{i} \geq t \mid \xi_{i}=1\right) \operatorname{Pr}\left(\xi_{i}=1\right) \\
& =1-p_{i}+p_{i} S\left(t \mid \xi_{i}=1\right)
\end{aligned}
$$

for $t<\infty$. Note that $S_{i}(t) \rightarrow\left(1-p_{i}\right)$ as $t \rightarrow \infty$, implying that the marginal survival function $S_{i}(t)$ tends to the cure probability $\left(1-p_{i}\right)$ for large $t$. As long as the incidence probability $p_{i}$ and the conditional survival function $S\left(t \mid \xi_{i}=1\right)$, or the conditional hazard function $h\left(t \mid \xi_{i}=1\right)$, are obtained, the marginal survival function $S_{i}(t)$ can be formed using (4). In what follows we discuss how to model $h\left(t \mid \xi_{i}=1\right)$ and $p_{i}$ in terms of covariates of interest. For simplicity, we assume the censoring mechanism is noninformative and is independent of the longitudinal response $\boldsymbol{Y}$.

Farewell [25] proposed to use the following logistic regression model

$$
\operatorname{Pr}\left(\xi_{i}=1 \mid z_{i}\right)=\frac{\exp \left(\boldsymbol{z}_{i}^{\prime} \boldsymbol{\lambda}\right)}{1+\exp \left(\boldsymbol{z}_{i}^{\prime} \boldsymbol{\lambda}\right)}
$$

to model the incidence probability $p_{i}$, where $\boldsymbol{z}_{i}$ and $\boldsymbol{\lambda}$ are covariates and parameters, respectively. He also suggested a parametric survival model for 
$S(t \mid \xi=1)$. Sy and Taylor [16] proposed the proportional hazard cure model

$$
h_{i}\left(t \mid \xi_{i}=1, \boldsymbol{w}_{i}\right)=h_{0}\left(t \mid \xi_{i}=1\right) \exp \left(\boldsymbol{w}_{i}^{\prime} \boldsymbol{\alpha}\right),
$$

where $\boldsymbol{w}_{i}$ and $\boldsymbol{\alpha}$ are covariates and parameters, respectively, and $h_{0}\left(t \mid \xi_{i}=1\right)$ is the conditional baseline hazard function. This model is not designed for cases where longitudinal data exist.

The models above, however, take no account of the likely relation among the incidence probability, the conditional hazard function and the tumour volume $\boldsymbol{Y}$. As a result, the separate use of those models may lead to biased statistical inferences. Instead, we propose to use the random effects $\boldsymbol{u}_{i}$ in the longitudinal model (1), to link the longitudinal and survival-cure models. In other words, we use the following logistic regression model

$$
\operatorname{Pr}\left(\xi_{i}=1 \mid \boldsymbol{z}_{i}, \boldsymbol{u}_{i}\right)=\frac{\exp \left(\boldsymbol{z}_{i}^{\prime} \boldsymbol{\lambda}+\boldsymbol{\pi}_{2}^{\prime} \boldsymbol{u}_{i}\right)}{1+\exp \left(\boldsymbol{z}_{i}^{\prime} \boldsymbol{\lambda}+\boldsymbol{\pi}_{2}^{\prime} \boldsymbol{u}_{i}\right)}
$$

to model the incidence probability, and use the following proportional hazard frailty model (see [7])

$h_{i}\left(t \mid \xi_{i}=1, \boldsymbol{u}_{i}, \boldsymbol{w}\right)=h_{0}\left(t \mid \xi_{i}=1\right) \exp \left(\boldsymbol{w}_{i}^{\prime}(t) \boldsymbol{\alpha}+\pi_{11}\left(u_{i 0}+u_{i 1} t\right)+\pi_{12} u_{i 0}+\pi_{13} u_{i 1}\right)$

to model the conditional hazard function, where $\boldsymbol{\pi}_{1}=\left(\pi_{11}, \pi_{12}, \pi_{13}\right)$ in (5) and $\boldsymbol{\pi}_{2}=\left(\pi_{21}, \pi_{22}\right)^{\prime}$ in (4) are unknown link parameters. If the estimators of $\boldsymbol{\pi}_{1}$ and $\boldsymbol{\pi}_{2}$ are statistically significant, we conclude that the joint modelling of the longitudinal and survival-cure data is necessary. Otherwise, the separate 
modelling strategy may be preferred.

Note that the incidence probability in (4) considered here is the probability that the incidence event eventually occurs. Therefore it does not depend on time. In our model the incidence probability depends on $\boldsymbol{z}_{i}$ (e.g. treatments) and $\boldsymbol{u}_{i}$ (the tumor characteristics: baseline effect $u_{i 0}$ and rate effect $u_{i 1}$ ). We may consider that the incidence probability depends on time. This may be done by using a competing risk model or a Markov transition model to model the hazard rates for death event and cure event. But in the study the cure event time was not observed exactly, and hence here we only consider the simple model (4). Although a mouse whose tumor size is less than 0.01 at week 12 was considered as cured, it is not clear when exactly the mouse's tumor disappears due to the constraint of the tumor size reading machine.

\subsection{Complete log-likelihood function}

Given random effect $\boldsymbol{u}_{i}$, we assume the longitudinal data and survival-cure data are independent. It is noted that the incidence $\xi_{i}$ may be observable or unobservable, depending on whether or not the censoring occurs. Define $\xi=\left\{\xi^{o}, \xi^{m}\right\}$ as the set of all the incidences $\xi_{i}$ 's, where $\xi^{o}$ and $\xi^{m}$ are the collections of the observable and unobservable incidences, respec-

tively. The observed data are then $\mathcal{D}=\left\{\left(Y_{i}, T_{i}, \delta_{i}, X_{i}, w_{i}, z_{i}\right): i=1, \ldots, m\right\}$ and $\xi^{o}$. Then the complete log-likelihood function of the parameters $\Theta=$ $\left(\boldsymbol{\beta}, \boldsymbol{\alpha}, \boldsymbol{\lambda}, \sigma_{\varepsilon}^{2}, \Sigma_{u}^{2}, \boldsymbol{\psi}, \boldsymbol{\pi}_{1}, \boldsymbol{\pi}_{2}, h_{0}\right)$ and the unobservable data $\left(\boldsymbol{u}, \xi^{m}\right)$, apart from 
a constant, can be written as

$$
\begin{aligned}
& \ell\left(\Theta, \boldsymbol{u}, \xi^{m} \mid \mathcal{D}, \xi^{o}\right) \\
= & -\frac{N}{2} \log \sigma_{\varepsilon}^{2}-\frac{1}{2 \sigma_{\varepsilon}^{2}} \sum_{i=1}^{m} \sum_{j=1}^{n_{i}}\left(Y_{i}\left(t_{j}\right)-\psi_{0}-\psi_{1} t_{j}-\psi_{2} t_{j}^{2}-\boldsymbol{X}_{i} \boldsymbol{\beta}-u_{i 0}-u_{i 1} t_{j}\right)^{2} \\
+ & \sum_{i=1}^{m}\left\{\delta_{i} \xi_{i}\left(\log h_{0}\left(\tilde{T}_{i} \mid \xi_{i}=1\right)+\boldsymbol{w}_{i}^{\prime}\left(\tilde{T}_{i}\right) \boldsymbol{\alpha}+\pi_{11}\left(u_{i 0}+u_{i 1} \tilde{T}_{i}\right)+\pi_{12} u_{i 0}+\pi_{13} u_{i 1}\right)\right. \\
- & \left.\xi_{i} \int_{0}^{\tilde{T}_{i}} h_{0}\left(t \mid \xi_{i}=1\right) \exp \left(\boldsymbol{w}_{i}^{\prime}(t) \boldsymbol{\alpha}+\pi_{11}\left(u_{i 0}+u_{i 1} t\right)+\pi_{12} u_{i 0}+\pi_{13} u_{i 1}\right) d t\right\}-\frac{m}{2} \log \left|\Sigma_{u}\right| \\
+ & \sum_{i=1}^{m}\left\{\xi_{i}\left(\boldsymbol{z}_{i}^{\prime} \boldsymbol{\lambda}+\pi_{2} \boldsymbol{u}_{i}\right)-\log \left(1+\exp \left(\boldsymbol{z}_{i}^{\prime} \boldsymbol{\lambda}+\pi_{2} \boldsymbol{u}_{i}\right)\right)\right\}-\frac{1}{2} \boldsymbol{u}_{i}^{\prime} \Sigma_{u}^{-1} \boldsymbol{u}_{i} .
\end{aligned}
$$

Note that for the above log-likelihood function, the domain for $\boldsymbol{\psi}$ is $\boldsymbol{\Psi}$ defined in Condition 1, the intrinsic condition. Therefore, under the intrinsic condition, the log-likelihood will be $-\infty$, if the $\boldsymbol{\psi} \notin \Psi$; the log-likelihood is given by the above formula (6) if $\boldsymbol{\psi} \in \boldsymbol{\Psi}$.

For the baseline hazard function $h_{0}\left(t \mid \xi_{i}=1\right)$, we assume it is a piecewise constant function as well, that is,

$$
h_{0}\left(t \mid \xi_{i}=1\right)=h_{0 k}, \quad \text { for } t_{k-1} \leq t<t_{k} \quad\left(k=1, \cdots, n_{h}\right),
$$

where $\boldsymbol{h}_{0}=\left(h_{01}, \cdots, h_{0 n_{h}}\right)^{\prime}$ are unknown parameters and $n_{h}$ is not greater than $n$.

In our study $\boldsymbol{w}_{i}^{\prime}(t)$ is chosen as the cumulative dose level until time $t$ and it is thus a piecewise constant. Therefore the evaluation for the integration in (6) is straightforward. Even if there is a dependence on $t^{2}$ in the definite 
integration of (6), it can still be evaluated numerically by using the command 'integrate' in the R package. Therefore this will not limit the applicability of our model.

\section{Bayesian Inference}

We propose to use a Bayesian approach to make statistical inferences for the joint models (1), (4) and (5) to avoid the analytical intractable integral problem involved in the marginalized log-likelihood function. Markov chain Monte Carlo (MCMC) is applied in our implementation. Rather than integrating out the random effects $\boldsymbol{u}_{i}$ and the missing values $\xi^{m}$ from (6), we sample $\mathbf{u}$ and $\xi^{m}$, as well as other parameters, from their corresponding conditional posterior distributions.

We specify independent normal priors for the parameters $\beta, \alpha$ and $\lambda$, of which all are assumed to have very large variances. We also specify inverse Gamma and inverse Wishart priors for the random errors variance $\sigma_{\varepsilon}^{2}$ and random effects variance $\Sigma_{u}$, respectively. We choose a Gamma prior for each $h_{0 j}(j=1,2, \ldots, n)$, so that a conjugate posterior distribution for $h_{0 j}$ is easy to obtain. We assume a normal distribution, $N(\tau, \varsigma)$, as the prior for each component of the link parameters $\boldsymbol{\pi}_{1}$ and $\boldsymbol{\pi}_{2}$.

We propose to use the DIC value to select the most appropriate model. The DIC value consists of two terms, one for goodness-of-fit measured by the deviance evaluated at the posterior mean of parameters, and the other ac- 
counting for a penalty defined by twice of the effective number of parameters. The latter is defined by the mean deviance minus the deviance evaluated at the posterior mean. Under the model assumption with missing data, the DIC is defined by

$\left.\left.D I C=-4 E_{\Theta, \boldsymbol{u}, \xi^{m}}\left[\ell\left(\Theta, \boldsymbol{u}, \xi^{m} \mid \mathcal{D}, \xi^{o}\right) \mid \mathcal{D}, \xi^{o}\right)\right]+2 E_{\boldsymbol{u}, \xi^{m}}\left[\ell\left(\tilde{\Theta}, \boldsymbol{u}, \xi^{m} \mid \mathcal{D}, \xi^{o}\right) \mid \mathcal{D}, \xi^{o}\right)\right]$

where $\tilde{\Theta}=E\left[\Theta \mid \mathcal{D}, U, \xi^{m}, \xi^{o}\right]$. See [27] and [28] for more details.

\section{Real data analysis}

Following the notation in Section 2, we denote $n_{i}$ as the number of repeated measurements for the $i$ th mouse $\left(n_{i} \leq 12\right)$. Let $x_{i}^{(1)}(t)$ be the cumulative dose of TMZ and $x_{i}^{(2)}(t)$ be the cumulative dose of CPT-11, which are received by the $i$ th mouse till week $t\left(t=1, \ldots, n_{i}, i=1, \ldots, 51\right)$. To account for the

synergism of the two drugs, following [1] we take $x_{i}^{(3)}(t)=\sqrt{x_{i}^{(1)}(t) x_{i}^{(2)}(t)}$ as the interaction term. Let $\boldsymbol{X}_{i}(t)=\left(x_{i}^{(1)}(t), x_{i}^{(2)}(t), x_{i}^{(3)}(t)\right)^{\prime}$. The longitudinal sub-model in (1) is then used to model the activity of the TMZ combined with CPT-11 against Rh18 tumour growth for the $i$ th mouse in the xenograft experiments. In our data analysis studies, we rescale time points as $t_{j}=t_{j} / 10$ and the dose level of TMZ divided by 100 .

Among the 51 mice, in total 25 mice died of toxicity or were sacrificed as the tumour volumes were quadrupled. On the other hand, 14 mice were considered to be cured by the end of the experiment, as they survived 12 
months and have tumor size smaller than 0.01 . The remaining 12 mice were not cured by the end of the experiment but survived longer than 12 week, so that their true lifetimes and cure incidence indicators are not observable. We then use the survival model (5) to model the conditional hazard function $h_{i}\left(t \mid \xi_{i}=1, \boldsymbol{w}_{i}(t), \boldsymbol{u}_{i}\right)$, where $\boldsymbol{w}_{i}(t)=\boldsymbol{X}_{i}(t-)$ is the value of $\boldsymbol{X}_{i}$ right before the time point $t$. The baseline hazard rate $h_{0}\left(t \mid \xi_{i}=1\right)$ is assumed to be a piecewise constant function

$$
h_{0}\left(t \mid \xi_{i}=1\right)=h_{0 j}, t_{j-1} \leq t<t_{j},\left(j=1, \cdot, n_{h}\right)
$$

where we choose $n_{h}=4$ because in the 12 -week period of experiment the treatments are given to mice as a cycle of every three weeks (see Table 1 in the supplementary file).

We also use the model (4) to model the incidence probability $\operatorname{Pr}\left(\xi_{i}=\right.$ $\left.1 \mid \boldsymbol{z}_{i}, \boldsymbol{u}_{i}\right)$, where $\boldsymbol{z}_{i}=\left(z_{i}^{(1)}, z_{i}^{(2)}, z_{i}^{(3)}, 1\right)^{\prime}$ in which $z_{i}^{(1)}$ and $z_{i}^{(2)}$ are the average weekly-dose levels of TMZ and CPT-11 for subject $i$, respectively, and $z_{i}^{(3)}$ is the associated interaction term. Note that we use the weekly-average dose levels $z_{i}^{(s)}$ rather than the cumulative dose $x_{i}^{(s)}(t)(s=1,2,3)$ as the covariates for modeling of the incidence probability $\operatorname{Pr}\left(\xi_{i}=1 \mid \boldsymbol{z}_{i}, \boldsymbol{u}_{i}\right)$. The reason is that our aim is to model how the incidence probability $\operatorname{Pr}\left(\xi_{i}=1 \mid \boldsymbol{z}_{i}, \boldsymbol{u}_{i}\right)$ depends on treatments rather than time or time-related variables such as the cumulative dose $x_{i}^{(s)}(t)$. It is noted that different weekly-average dose level is taken as a different treatment here. Of course, it may be of interests to see how the incidence probability is related to the tumor volume that changes 
over time. In this case, the model for the incidence probability is a dynamic model and the latent Markov transition model proposed by [29] may be a key to resolve the issue. We leave this as future work.

The parameters of interest in the survival-cure models are the fixed effects parameters $\alpha=\left(\alpha_{1}, \alpha_{2}, \alpha_{3}\right)^{\prime}, \lambda=\left(\lambda_{1}, \lambda_{2}, \lambda_{3}, \lambda_{4}\right)^{\prime}$ and the link parameters $\boldsymbol{\pi}_{1}$ and $\boldsymbol{\pi}_{2}$.

Following [21], the prior for each element of $\boldsymbol{\beta}, \boldsymbol{\alpha}$ and $\boldsymbol{\lambda}$ is chosen to be $N(0,10000)$. The priors for $\sigma_{\epsilon}^{-2}$ and each element of $h_{0}$ are all chosen to be Gamma $(0.001,0.001)$. The priors for $\Sigma_{u}$ is taken to be the inverse Wishart distribution with parameters $\left(4, I_{2}\right)$. For each element of the link parameters $\boldsymbol{\pi}_{1}, \boldsymbol{\pi}_{2}$, a non-informative normal prior $N(0,100)$ is applied.

To see if the model links are really necessary, we consider the following four possible link scenarios.

Case 1: longitudinal and survival-cure models are linked by $\boldsymbol{\pi}_{1}$ and $\boldsymbol{\pi}_{2}$.

Case 2: longitudinal and survival models are linked by $\boldsymbol{\pi}_{1}$ (i.e. $\boldsymbol{\pi}_{2}=\mathbf{0}$ ).

Case 3: longitudinal and cure models are linked by $\boldsymbol{\pi}_{2}$ (i.e. $\left.\boldsymbol{\pi}_{1}=\mathbf{0}\right)$.

Case 4: neither of these models are linked (i.e. $\boldsymbol{\pi}_{1}=\mathbf{0}, \boldsymbol{\pi}_{2}=\mathbf{0}$ ).

In Table 1 we summarize the posterior means and $95 \%$ credit intervals of the parameters in the models for each case above. We also provide the relative DIC values for all the four possible scenarios. In each case, we uses 10,000 iterations of MCMC sampling chains following a 5,000-iteration 'burn-in' period. 
[Table 1 about here.]

From Table 1, it turns out that the smallest DIC value is achieved by the model that links all the three sub-models, implying that the joint modelling may be necessary. The TMZ, the CPT-11 treatments and their interaction all have significant negative effects on the tumour growth, implying the treatments work in reducing the tumour growth. For the incidence model, we can see that the two treatments and their interaction have no significant effects on the incidence probability. The only significant estimate is for the intercept parameter $\lambda_{4}$, which is about 3.101 , leading to the incidence probability being $96 \%$ in the absence of treatments. In other words, without treatments the tumour-beard mice have only $4 \%$ chances to be cured. The parameters $\lambda_{2}=-2.799$ and $\lambda_{3}=-1.884$ are not significant, but their large negative values also suggest CPT-11 and the interaction of TMZ and CPT-11 may have effects on the incidence probability, i.e. treatments will increase the probability of cure. For the survival model, the CPT-11 has a significant positive effect on the survival time, but and TMZ-11 and the interaction of the two treatments are not significant.

For the model that links all the three sub-models, the estimate for the link parameter $\pi_{11}=0.141$ is significant. This implies that individual tumor departure from the mean tumor size is a significant risk factor for survivals. The link parameter $\pi_{11}$ is positive, showing that the bigger the Rh18 tumour volume departure from the mean tumor size the higher the hazard rate of death. The link parameter $\pi_{12}, \pi_{13}$ are not significant, showing that the 
individual departures from the intercept and from the slope do not have effects on survival. For the link parameter $\boldsymbol{\pi}_{2}=\left(\pi_{21}, \pi_{22}\right)$, the estimate for $\pi_{22}$ is significant, but the estimate for $\pi_{21}$ is not significant. This implies that the gradient $u_{i 1}$ (the rate of individual tumor growth) is a significant risk factor for the incidence probability. The higher the individual tumor growth rate, the lower the cure probability. Note that, for the full model (case 1), the random slope is the only significant predictor of cure.

When only having the link for the longitudinal and survival sub-models (case 2 in Table 1), all the link parameters of $\boldsymbol{\pi}_{1}$ are not significant, which implies that the tumor growth does not have significant effects on survival. This conflicts with reality. In addition, without having the second link parameter $\boldsymbol{\pi}_{2}$, the parameter vector $\lambda_{2}$ becomes significant (cases 2 and 4 ). The main reason for this is that the random effects are not considered in cases 2 and 4, which distorts the estimate of the fixed-effect parameter vector $\boldsymbol{\lambda}$.

When only having the link parameter $\boldsymbol{\pi}_{2}$ (case 3 in Table 1 ), the link parameter $\pi_{22}$ is also significant. Although most results in case 3 are similar to those in case 1, without having the first link parameter (case 3) has a smaller estimate for $\left|\alpha_{2}\right|=0.128$ than $\left|\alpha_{2}\right|=0.257$ in case 1 . Without the first link parameter, we may underestimate the effects of the treatment agent CPT-11, which is the only significant risk factor for survivals. For this reason and the DIC values, we suggest that case 1 , the full model, is more suitable for the data.

We also considered the models without using the intrinsic growth constraint. 
The results for the full model and separated models are provided in the supplementary materials. Basically the results of having the intrinsic growth and without having the intrinsic growth are consistent. The main difference is as follows. Without having the constraint, the baseline tumor volumes (i.e. no treatment) is estimated by $\phi_{2} t^{2}+\phi_{1} t+\phi_{0}$, with $\phi_{2}=5.598, \phi_{1}=-3.624$ and $\phi_{0}=-0.187$. This implies that without treatment, the tumor will decrease approximately before time 0.3 (week 3 as we use week divided by 10 for the new time scale), and then increase after that. However, this is not correct in practice, since the tumor will surely increase if no treatments are received. With having the constraint, the baseline tumor volume is estimated as $3.869 t^{2}+0.158 t-0.561$, an increasing function of time $t$.

\section{Simulation studies}

We mimic the real data in Section 4 in the following simulation studies. The covariates $\boldsymbol{X}_{i}(t), \boldsymbol{w}_{i}(t)$ and $\boldsymbol{z}_{i}$ are the same as that in the real data study. In other words, we have three covariates in the simulation: two treatments TMZ and CPT-11, and their interaction. These covariates are included in both the longitudinal and survival models. We also choose two dose levels for the TMZ $42 \mathrm{mg} / \mathrm{kg}$ and the CPT-11 $0.61 \mathrm{mg} / \mathrm{kg}$ and then consider eight groups of treatments as that in data analysis. The sample size is $m=51$ and the number of samples in each group is the same as that in the real data study. The independent random effects $\boldsymbol{u}_{i}$ are assumed to follow Normal distribution $N_{2}\left(0, \Sigma_{u}\right)$, the independent random errors $\varepsilon_{i j}$ follow Normal 
distribution $N\left(0, \sigma_{\varepsilon}^{2}\right)$, and $\boldsymbol{u}_{i}$ and $\varepsilon_{i j}$ are mutually independent.

Given the random effects $\boldsymbol{u}_{i}$ and covariates $\boldsymbol{z}_{i}$, the incidence event $\xi_{i}$ is generated from the model (4).

For those subjects with incidence indicator $\xi_{i}=1$, on the other hand, the survival model (5) is used to generate the survival outcomes $T_{i}$. To mimic the data, the baseline hazard rate $h_{0}\left(t \mid \xi_{i}=1\right)$ is assumed to be a piecewise constant function

$$
h_{0}\left(t \mid \xi_{i}=1\right)=h_{0 j}, t_{j-1} \leq t<t_{j},(j=1, \cdots, n)
$$

and $h_{01}=1.6, h_{02}=3.0, h_{03}=9.8, h_{04}=16.7$, which are similar to the estimated values in the real data analysis. It is assumed that the baseline hazard has an increasing jump because the hazard rate increases in the absence of treatments.

Our data are generated from the full model, with linking parameters $\pi_{11}=$ $0.6, \pi_{12}=0.7, \pi_{13}=0.0, \pi_{21}=0$ and $\pi_{22}=1.22$.

We compare the simulation results for two models, the model with the association $\left(\boldsymbol{\pi}_{1} \neq \mathbf{0}\right.$ and $\left.\boldsymbol{\pi}_{2} \neq \mathbf{0}\right)$ and the model without the association $\left(\boldsymbol{\pi}_{1}=\mathbf{0}, \boldsymbol{\pi}_{2}=\mathbf{0}\right)$. We simulate 100 data sets and calculate the sample mean and the sample standard deviation for each parameter estimator. For each data set, we draw 5,000 random samples from the posterior distributions following a 5,000-iteration 'burn-in' period in order to estimate the parameters. Table 2 provides the parameter estimators, standard deviations and 
coverage probability of credible interval.

From Table 2, we can see that the joint models and the separated modelling give similar parameter estimates for the longitudinal sub-model. When the latent association of longitudinal and survival-cure data does exist, i.e., $\pi_{11} \neq 0, \pi_{12} \neq 0$ and $\pi_{22} \neq 0$, the proposed joint modelling approach per-

forms well. For example, the link parameter estimators of $\pi_{11}, \pi_{12}$ and $\pi_{22}$ are statistically significant and very close to the true parameter values, which correctly identifies that the joint models are really necessary. On the contrary, the separated modelling approach that ignores the existing inherent association gives estimates with larger bias for most of the parameter estimation, except for $\alpha_{2}$ and $\lambda_{2}$. In addition, the separate model has lower coverage probability for $\alpha_{1}$ and $\beta_{3}$. The two models give similar coverage probabilities for all other parameters. Overall, statistical inferences for the survival models using the joint modelling approach are more sensible and reliable than those using the separate modelling method.

[Table 2 about here.]

\section{Discussion}

In this paper we propose a joint modelling approach to account for the likely inherent association for longitudinal data and survival-cure outcomes. We propose to use common random effects to connect the different models. The approach is then used to analyze a real data set arising from tumour xenograft 
experiments. Bayesian inferences are obtained using an MCMC approach, showing the parameter estimators from the posteriors are robust against the priors of the link parameters. Our conclusion on the data analysis is mostly consistent with [24] but the inherent association of different types of data is taken into account so that more information is discovered. Simulation studies show that the proposed joint modelling approach produces very satisfactory parameter estimators.

Some further research needs to be studied when each mouse has multiple tumours. [21] described an example of preclinical studies evaluating the anti-tumour effects of exemestane and tamoxifen for postmenopausal breast cancer, in which each mouse received subcutaneous injections at two sites and developed four tumours in the process. It is anticipated that multivariate longitudinal responses and multi-dimensional random effects will be involved and additional correlation between tumours for the same mice should be accounted for. We will report this in a follow-up paper.

In our study, the cure probability is just the probability that a mouse is cured or not, eventually. It does not depend on time, which is a limitation of our work. In other studies, it may also be interesting to consider that the cure probability depends on time, if the cure times are available. A Markov transition model might be employed to deal with such problems. 


\section{References}

[1] Tan M., Fang H.B., Tian G.L. and Houghton P.J. (2005a). Repeated measures models with constrained parameters for incomplete data in tumour xenograft experiments. Statistics in Medicine, 24:109-119.

[2] Tan M., Fang H.B., Tian G.L. and Houghton P.J. (2002). Small sample inference for incomplete longitudianl data with truncation and censoring in tumour xenograft models. Biometrics, 58:612620.

[3] Fang H.B., Tian G.L. and Tan M. (2004). Hierarchical models for tumour xenograft experiments in drug development. Journal of Biopharmaceutical statistics, 14:931-945.

[4] Tsiatis A. and Davidian M. (2004). An overview of joint modelling of longitudinal and time-to-event data. Statistica Sinica, 14:809834.

[5] Tsiatis A., De Gruttola V. and Wulfsohn, M. (1995). Modeling the relationship of survival to longitudinal data measured with error: Applications to survival and CD4 counts in patients with AIDS. Journal of the American Statistical Association, 90:27-37.

[6] Wulfsohn M.S. and Tsiatis A.A. (1997). A joint model for survival and longitudinal data measured with error. Biometrics, 53:330339. 
[7] Henderson R., Diggle P. and Dobson A. (2000). Joint modelling of longitudinal measurements and event time data. Biostatistics, 1: 465-480.

[8] Song X., Davidian M. and Tsiatis A. A. (2002a). An estimator for the proportional hazards model with multiple longitudinal covariates measured with error. Biostatistics, 3:511-528.

[9] Song X., Davidian M. and Tsiatis A. A. (2002b). A semiparametric estimator for the proportional hazards model with longitudinal covariates measured with error. Biometrika, 88:447-458.

[10] Song X. and Huang Y. (2005). On corrected score approach for proportional hazards model with covariate measurement error. Biometrics, 61:702-714.

[11] Wang C. Y. (2006). Corrected score estimator for joint modelling of longitudinal and failure time data. Statistica Sinica, 16:235-253.

[12] Xu J. and Zeger S. (2001). Joint analysis of longitudinal data comprising repeated measures and times to events. Applied Statistics, 50:375-387.

[13] Elashoff R., Li G. and Li N. (2007). An approach to joint analysis of longitudinal measurements and competing risks failure time data. Statistics in Medicine, 26:2813-2835.

[14] Price D. L. and Manatunga A. K. (2001). Modelling survival 
data with a cured fraction using frailty models. STATISTICS IN MEDICINE, 20:1515-1527.

[15] Corbiere F., Commenges D., Taylor J. M. G. and Joly P. A penalized likelihood approach for mixture cure models. Statistics in Medicine, 28:510C524.

[16] Sy J.P. and Taylor J.M.G. (2000). Estimation in a Cox proportional hazards cure model. Biometrics, 56:227-236.

[17] Xiang L., Ma X. and Yau K.K.W. (2011). Mixture cure model with random effects for clustered interval-censored survival data. Statistics in Medicine, 30(9):995-1006.

[18] Brown E. R. and Ibrahim J. G. (2003). Bayesian approaches to joint cure-rate and longitudinal models with applications to cancer vaccine trials. Biometrics, 59(3):686-693.

[19] Yu M., Law N.J., Taylor J.M.G. and Sandler H.M. (2004) Joint longitudinal-survival-cure models and their application to prostate cancer. Statistic Sinica, 14:835-862.

[20] Yu M., Taylor J.M.G. and Sandler H.M. (2008) Individual Prediction in Prostate Cancer Studies Using a Joint Longitudinal Survival-Cure Model. Journal of the American Statistical Association, 103:178-186.

[21] Tan M., Fang H.B. and Tian G.L. (2005b). Statistical Analysis for Tumor Xenograft Experiments in Drug Development. Contem- 
porary Multivariate Analysis and Wxperimental Design-In Honor Celebration of Professor Kai-Tai Fang's 65th birthday, 351-368. The World Scientific Publisher.

[22] Laird N. M. and Ware J. H. (1982) Random-effects models for longitudinal data. Biometrics, 38(4):963-974.

[23] Liang H. and Sha N. (2004) Modeling antitumor activity by using a non-linear mixed-effects model. Mathematical Biosciences, 189:6173.

[24] Houghton P.J., Stewart C.F., Cheshire P.J., Richmond L.B., Kirstein M.N., Poquette C.A., Tan M., Friedman H.S. and Brent T.P. (2000). Antitumour activity of temozolomid combined with irinotecan is partly independent of $O^{6}$-methylguanine-DNA methyltransferase and mismatch repair phenotypes in xenograft models. Clinical Cancer Research, 6:4110-4118.

[25] Farewell V.T. (1982). The use of mixture models fo the analysis of survival data with long-term survivors. Biometrics, 38:1041-1046.

[26] Gilks W.R. and Wild P. (1992). Adaptive rejection sampling for Gibbs sampling. Applied Statistics, 41:337-348.

[27] Spiegelhalter D.J., Best N.G., Carlin B.P. and van der Linde A. (2002). Bayesian measures of model complexity and fit (with discussion). Journal of the Royal Statistical Society, Ser. B., 64:583639. 
[28] Celeux G., Forbes F., Robert C.P. and Titterington D. M. (2006). Deviance Information Criteria for Missing Data Models. Bayesian Analysis, 4:651-674.

[29] Reboussin B. A., Liang K-Y and Reboussin D. M. (1999). Estimating Equations for a Latent Transit ion Model with Multiple Discrete Indicators. biometrics, 55:839-845. 

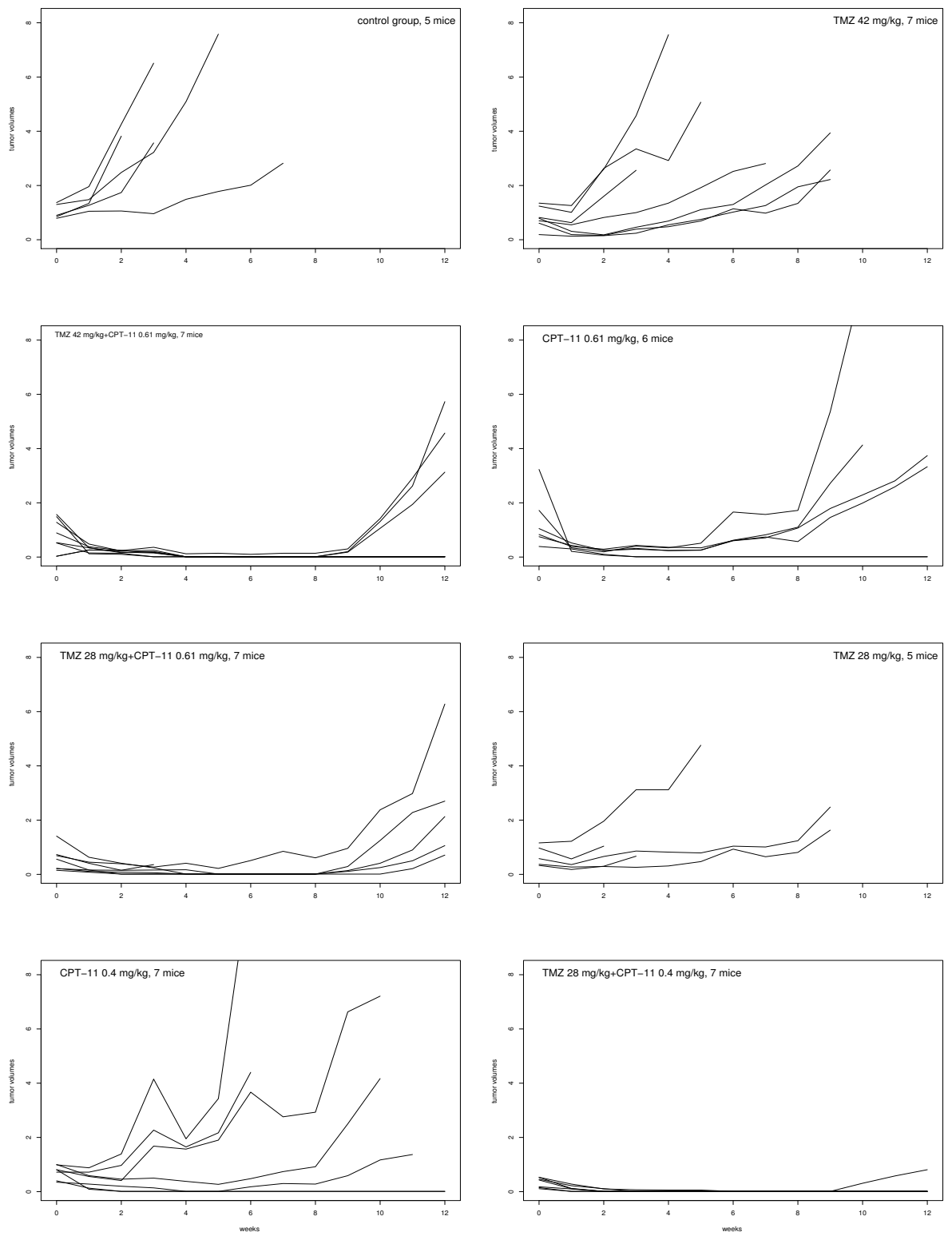

Figure 1: Observed tumor volumes for the eight treatment groups for 8 groups 
Table 1: Posterior mean (95\% credible interval) of the parameters and the DIC values. Parameter $\boldsymbol{\beta}$ corresponds to the covariate effects for the tumor sizes; parameter $\boldsymbol{\alpha}$ correspond to the covariate effects on the survival time; parameter $\boldsymbol{\lambda}$ correspond to the covariate effects on the incidence probability.

\begin{tabular}{|c|c|c|c|c|c|}
\hline & Par. & Case 1 & Case 2 & Case 3 & Case 4 \\
\hline & DIC & 477.1 & 518.3 & 530.7 & 555.4 \\
\hline \multirow{7}{*}{$\begin{array}{l}\text { Longitudinal } \\
\text { submodel }\end{array}$} & & & & & \\
\hline & $\psi_{0}$ & -0.561 & -0.442 & -0.227 & -0.484 \\
\hline & & $(-0.996,-0.232)$ & $(-0.755,-0.199)$ & $(-0.635,0.202)$ & $(-0.765,-0.164)$ \\
\hline & $\psi_{1}$ & 0.158 & 0.286 & 0.242 & 0.472 \\
\hline & & $(0.005,0.696)$ & $(0.008,0.829)$ & $(0.007,0.690)$ & $(0.014,1.241)$ \\
\hline & $\psi_{2}$ & 3.869 & 4.251 & 4.402 & 4.243 \\
\hline & & $(3.401,4.307)$ & $(3.655,4.946)$ & $(3.804,5.038)$ & $(3.560,4.901)$ \\
\hline \multicolumn{6}{|l|}{ Longitudinal } \\
\hline \multirow[t]{2}{*}{ TMZ } & $\beta_{1}$ & -0.289 & -0.272 & -0.248 & -0.237 \\
\hline & & $(-0.404,-0.177)$ & $(-0.399,-0.130)$ & $(-0.347,-0.136)$ & $(-0.401,-0.112)$ \\
\hline \multirow[t]{2}{*}{ CPT-11 } & $\beta_{2}$ & -0.253 & -0.236 & -0.231 & -0.256 \\
\hline & & $(-0.297,-0.206)$ & $(-0.280,-0.181)$ & $(-0.279,-0.194)$ & $(-0.318,-0.189)$ \\
\hline \multirow[t]{2}{*}{ Interaction } & $\beta_{3}$ & -0.070 & -0.069 & -0.128 & -0.052 \\
\hline & & $(-0.130,-0.016)$ & $(-0.112,-0.012)$ & $(-0.245,-0.052)$ & $(-0.144,-0.029)$ \\
\hline \multicolumn{6}{|l|}{ Survival } \\
\hline \multirow[t]{2}{*}{ TMZ } & $\alpha_{1}$ & -0.142 & -0.056 & -0.073 & -0.056 \\
\hline & & $(-0.461,0.198)$ & $(-0.297,0.167)$ & $(-0.285,0.126)$ & $(-0.267,0.146)$ \\
\hline \multirow[t]{2}{*}{ CPT-11 } & $\alpha_{2}$ & -0.257 & -0.119 & -0.128 & -0.118 \\
\hline & & $(-0.453,-0.092)$ & $(-0.218,-0.017)$ & $(-0.222,-0.039)$ & $(-0.210,-0.031)$ \\
\hline \multirow[t]{2}{*}{ Interaction } & $\alpha_{3}$ & 0.509 & 0.380 & 0.232 & 0.402 \\
\hline & & $(-0.322,1.380)$ & $(-0.090,0.740)$ & $(-0.299,0.665)$ & $(-0.010,0.741)$ \\
\hline \multicolumn{6}{|l|}{ Cure } \\
\hline \multirow[t]{2}{*}{$\mathrm{TMZ}$} & $\lambda_{1}$ & -0.368 & -0.241 & 0.092 & 0.018 \\
\hline & & $(-2.797,2.487)$ & $(-1.581,2.176)$ & $(-2.316,2.769)$ & $(-1.794,2.196)$ \\
\hline \multirow[t]{2}{*}{ CPT-11 } & $\lambda_{2}$ & -2.799 & $-2,289$ & -2.455 & -2.301 \\
\hline & & $(-5.513,0.667)$ & $(-4.138,-0.617)$ & $(-5.029,-0.068)$ & $(-4.263,-0.773)$ \\
\hline \multirow[t]{2}{*}{ Interaction } & $\lambda_{3}$ & -1.884 & -2.025 & -2.022 & -1.910 \\
\hline & & $(-5.2341 .696)$ & $(-4.427,0.266)$ & $(-5.184,1.243)$ & $(-4.663,0.577)$ \\
\hline \multirow[t]{2}{*}{ Constant } & $\lambda_{4}$ & 3.101 & 2.813 & 3.404 & 2.942 \\
\hline & & $(0.399,5.395)$ & $(0.720,4.007)$ & $(1.203,6.169)$ & $(1.051,5.036)$ \\
\hline \multicolumn{6}{|c|}{ Links between survival and longitudinal } \\
\hline \multirow[t]{2}{*}{$u_{i 0}+u_{i 1} t$} & $\pi_{11}$ & 0.141 & 0.035 & 0 & 0 \\
\hline & & $(0.033,0.295)$ & $(-0.177,0.378)$ & - & - \\
\hline \multirow[t]{2}{*}{$u_{i 0}$} & $\pi_{12}$ & 0.161 & 0.0152 & 0 & 0 \\
\hline & & $(-0.599,0.717)$ & $(-0.258,0.344)$ & - & - \\
\hline \multirow[t]{2}{*}{$u_{i 1}$} & $\pi_{13}$ & 0.092 & 0.003 & 0 & 0 \\
\hline & & $(-0.387,0.311)$ & $31(-0.235,0.202)$ & - & - \\
\hline \multicolumn{6}{|c|}{ Links between curel and longitudinal } \\
\hline \multirow[t]{2}{*}{$u_{i 0}$} & $\pi_{21}$ & -0.713 & 0 & -0.164 & 0 \\
\hline & & $(-4.672,2.044)$ & - & $(-2.801,1.684)$ & - \\
\hline$u_{i 1}$ & $\pi_{22}$ & 2.006 & 0 & 1.128 & 0 \\
\hline
\end{tabular}


Table 2: Simulation results for the case that there is a latent association, i.e., $\boldsymbol{\pi}_{1} \neq \mathbf{0}$ and $\boldsymbol{\pi}_{2} \neq \mathbf{0}$. The sample size $\mathrm{m}=51$.

Par. True Separate modelling Joint modelling Estimates St.D. Coverage prob Estimates St.D. Coverage

\begin{tabular}{cccccccc}
\hline$\psi_{0}$ & -0.40 & -0.395 & 0.038 & 0.95 & -0.382 & 0.040 & 0.96 \\
$\psi_{1}$ & 0.80 & 0.835 & 0.244 & 0.94 & 0.823 & 0.279 & 0.95 \\
$\psi_{2}$ & 3.60 & 3.587 & 0.170 & 0.95 & 3.604 & 0.168 & 0.96 \\
$\beta_{1}$ & -0.30 & -0.309 & 0.018 & 0.96 & -0.308 & 0.016 & 0.93 \\
$\beta_{2}$ & -0.27 & -0.273 & 0.010 & 0.90 & -0.273 & 0.010 & 0.90 \\
$\beta_{3}$ & -0.01 & -0.015 & 0.012 & 0.85 & -0.008 & 0.012 & 0.90 \\
\hline$\alpha_{1}$ & 0 & -0.184 & 0.140 & 0.88 & -0.171 & 0.139 & 0.90 \\
$\alpha_{2}$ & -0.22 & -0.220 & 0.054 & 0.96 & -0.236 & 0.077 & 0.96 \\
$\alpha_{3}$ & 0 & 0.135 & 0.122 & 0.93 & 0.116 & 0.155 & 0.94 \\
\hline$\lambda_{1}$ & 0 & 0.759 & 0.658 & 0.90 & 0.701 & 0.772 & 0.93 \\
$\lambda_{2}$ & 0 & -0.164 & 0.531 & 0.97 & -0.184 & 0.552 & 0.97 \\
$\lambda_{3}$ & 0 & -0.070 & 0.362 & 0.95 & -0.060 & 0.334 & 0.97 \\
$\lambda_{4}$ & 3.35 & 4.154 & 0.881 & 0.91 & 4.015 & 0.960 & 0.91 \\
\hline$\pi_{11}$ & 0.6 & - & - & - & 0.544 & 0.206 & 0.95 \\
$\pi_{12}$ & 0.7 & - & - & - & 0.688 & 0.201 & 0.96 \\
$\pi_{13}$ & 0.0 & - & - & - & 0.056 & 0.125 & 0.94 \\
$\pi_{21}$ & 0.0 & - & - & - & -0.055 & 0.197 & 0.96 \\
$\pi_{22}$ & 1.22 & - & - & - & 1.186 & 0.173 & 0.96 \\
\hline \hline
\end{tabular}

